\title{
Gait Analysis and Knee Kinematics in Patients with Anterior Cruciate Ligament Rupture: Before and After Reconstruction
}

\author{
Dmitry Skvortsov ${ }^{1,2,3, *(0)}$, Sergey Kaurkin ${ }^{1,2}$, Alexander Akhpashev ${ }^{2,4}$, Aljona Altukhova ${ }^{2}$, \\ Alexander Troitskiy ${ }^{2}$ and Nikolay Zagorodniy ${ }^{3}$ \\ 1 Rehabilitology and Sport medicine Department, Pirogov Russian National Research Medical \\ University (RNRMU), ul. Ostrovitianova, 1, Moscow 117513, Russia; kaurkins@bk.ru \\ 2 Federal Research and Clinical Centre of Russia's Federal Medical-Biological Agency (FNKC FMBA), \\ Orekhoviy bulvar, 28, Moscow 115682, Russia; akhpashev@gmail.com (A.A.); altukhova.aa@bk.ru (A.A.); \\ administracia83@bk.ru (A.T.) \\ 3 National Medical Research Center of Traumatology and Orthopedics N.N. Priorova, Volga research medical \\ University; pl. Minin and Pozharsky, 10/1, Nizhny Novgorod 603005, Russia; zagorodniy51@mail.ru \\ 4 Department of Traumatology and Orthopedics, Academy of Postgraduate Education at FNKC FMBA RF; \\ Peoples'FriendshipUniversity of Russia, Moscow 117198, Russia \\ * Correspondence: skvorcovdv@cito-priorov.ru; Tel.: +7-916-692-5419
}

Received: 20 March 2020; Accepted: 11 May 2020; Published: 13 May 2020

Featured Application: Authors are encouraged to provide a concise description of the specific application or a potential application of the work. This section is not mandatory.

\begin{abstract}
The objective of the study was to evaluate the clinical, functional, and biomechanical symptoms in patients with anterior cruciate ligament (ACL) rupture before and after ACL reconstruction. The study enrolled 20 patients and 20 healthy subjects as controls. Walking biomechanics was assessed at three time points: before surgery and three months and a year or more after surgical reconstruction. Impact loads on both sides differed significantly from the respective values before surgery $(\mathrm{p}<0.05)$. Walking cycle duration decreased with time after surgery. On both sides (affected and unaffected), hip movement amplitudes were significantly smaller than in control $(\mathrm{p}<0.05)$. They remained so in the follow-up periods after the reconstruction. Before ACL reconstruction, the amplitude of the main flexion of the knee was significantly reduced both on the affected and unaffected sides. The amplitude gradually increased after the reconstruction, and a year post-surgery, it reached, on the operated side, the same values as in the control group. Complete functional recovery of the knee joint was not achieved within a year after the ACL surgical reconstruction. The remaining changes, however, were not clinically pronounced and could only be detected by instrumental gait analysis. The compensatory processes developed bilaterally, in both the hip and knee joints.
\end{abstract}

Keywords: anterior cruciate ligament; biomechanics of walking; rehabilitation

\section{Introduction}

A considerable amount of literature has been published on the effects of anterior cruciate ligament (ACL) rupture on gait biomechanics. The authors describe different functional dynamics over time after surgery [1-3]. In their study, Goetschius et al. observed decreased amplitudes of knee flexion-extension-adduction and hip adduction in the affected limb 1.5 years after ACL reconstruction. At later periods (within 3.5 years), the authors found no differences from the contralateral limb, 
although they noted decreased amplitudes of knee flexion and knee and hip adduction 8.5 years after the ACL reconstruction [4]. Majewska J. et al. studied changes in knee joint kinematics before and six months after ACL reconstruction. The study showed a gradual functional improvement in the postoperative period-a significant increase in mean walking speed and in the movement amplitudes of both hips and knees, as well as improved range of ankle motion in the operated limb [5]. A study by Erhart-Hledik et al. also demonstrated improvement in some gait parameters over a period from two to eight years after ACL reconstruction. The authors noted an increasing angle of knee flexion and decreasing amplitudes of rotation and abduction of the operated limb [6].

On the other hand, some studies found no favorable functional changes [7]. For instance, Milandri et al. examined patients long after (approximately five years) ACL reconstruction. The study showed a decrease in the knee flexion angle on the affected side compared to control, both in walking and in running. In addition, maximum muscle strength asymmetry during running was greater for the unaffected limb [8]. Another study, by Shi et al., identified a relationship between the quadriceps strength asymmetry and the asymmetries in the lower extremity biomechanics during walking in patients after ACL reconstruction. The isometric quadriceps strength of the injured leg was significantly lower, and the flexion-extension amplitudes in the operated knee were smaller that those in the uninjured leg [9].

Despite the available literature on the effects of anterior cruciate ligament rupture on gait parameters, the dynamics of evolving changes is still poorly studied, and the available data are contradictory. This hampers rehabilitation and prediction of long-term outcomes.

The objective of the study was to evaluate the clinical, functional and biomechanical symptoms in patients with anterior cruciate ligament rupture before ACL reconstruction, 2-3 months after reconstruction, and in the long term.

\section{Materials and Methods}

The study enrolled 20 patients (10 males and 10 females) with MRI-confirmed complete ACL rupture and 20 healthy subjects as control (Table 1). The mean age of the patients was 34.2 years, ranging from 16 to 56 years. The clinical manifestations included seven cases of instability alone, seven cases of instability and discomfort, and six cases of pain. Instability was present in a total of 14 cases. The mean time from knee joint injury to ACL surgical reconstruction was five months.

Table 1. Subject characteristics.

\begin{tabular}{cccccc}
\hline Parameter & Assessment & $\begin{array}{c}\text { Number of } \\
\text { Assessed Subjects }\end{array}$ & $\begin{array}{c}\text { Time Point of } \\
\text { Assessment }\end{array}$ & Male/Female & $\begin{array}{c}\text { Mean Age, } \\
\text { Years }\end{array}$ \\
\cline { 2 - 6 } Patients & 1 & 20 & Before surgery & $10 / 10$ & 34.2 \\
\cline { 2 - 6 } & 2 & 20 & $\begin{array}{c}\text { Three months } \\
\text { after surgery }\end{array}$ & $10 / 10$ & 34.2 \\
\hline $\begin{array}{c}\text { Healthy } \\
\text { controls }\end{array}$ & 3 & 12 & $\begin{array}{c}\text { 12 months after } \\
\text { surgery }\end{array}$ & $7 / 5$ & 35.2 \\
\hline
\end{tabular}

Promptly after injury, the knee joint was immobilized for a period of 1-3 weeks (depending on the injury severity and concomitant disorders). After the surgical reconstruction, a hinged knee orthosis was applied. Rehabilitation included knee flexion to 90 degrees (later adding full flexion, for two weeks) and walking with additional support from the first days after surgery, followed by expansion of the motion regimen and removal of the orthosis. Exercise therapy sessions also started from the first day after surgery.

The control group included 20 healthy adult subjects ( 14 males and 6 females). Their mean age was 29.7 years. The study was conducted according to the principles of the Declaration of Helsinki 
and was approved by the local ethics committee. All subjects gave a written informed consent before the tests.

Gait biomechanics was assessed at three time points: at the initial consultation (before surgery) and then three months and a year or more after the surgical reconstruction. The first two assessments were done for all 20 patients, and the last one for only 12 patients. In healthy subjects, gait biomechanics was assessed only once.

The rehabilitation exercises were recommended on an individual basis for each patient. In the pre-operative period, they were strength exercises for both legs. In the post-operative period, rehabilitation included 2-3 weeks of individual sessions with a kinesiotherapist and a home exercise program. The sessions included manual kinesiotherapy, workout on special movement therapy devices, and muscle stimulation. The rehabilitation program was adjusted after the second assessment of walking biomechanics.

Biomechanical gait analysis was done using a procedure we had described earlier in reference [10]. The procedure was the same for all the subjects (patients and healthy controls). The kinematic parameters of the lower limb joints during walking were measured using inertial sensors. The sensors help evaluate the spatial characteristics of each movement in three mutually perpendicular planes. The recorded parameters included gait cycle time, hip and knee joint movements in three mutually perpendicular planes, and impact loads during walking. In this study, we used a human movement analysis system with five inertial sensors (Figure 1).

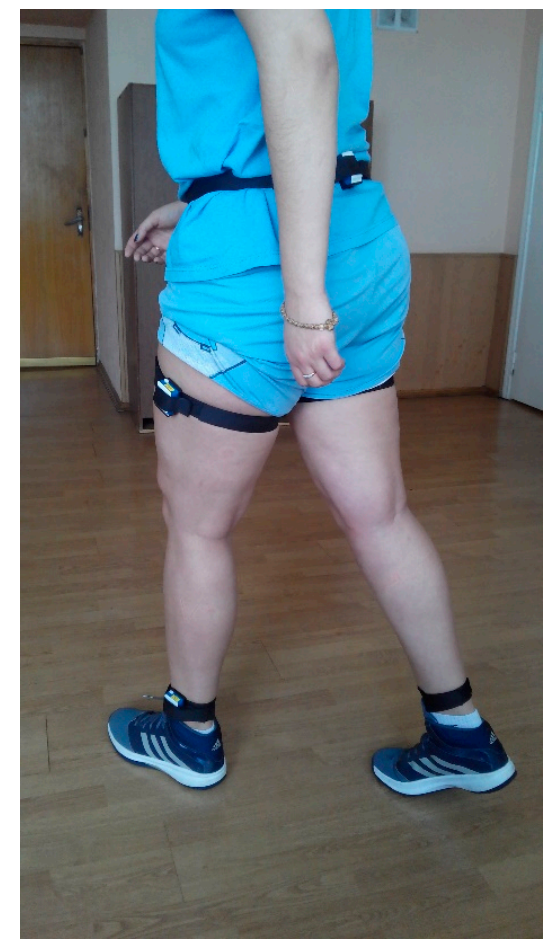

Figure 1. Placement of the sensors.

The sensors were fixed with special cuffs to the lower back, the lower third of the thigh and the lower third of the shank of the right and left legs. The movements and temporal characteristics were recorded while the subject was first walking 10 meters at an arbitrary pace. The walking trial was repeated 2-4 times, as appropriate. For the subsequent analysis, gait cycles were determined from the accelerometer data, whereupon mean goniograms were calculated for movements in the joints over the gait cycle and the temporal parameters of the gait cycle. Thus, we obtained goniograms of hip and knee motions in three mutually perpendicular planes and vertical acceleration curves for the shin-fixed sensors. 
The following temporal parameters were determined: walking cycle duration (WC), in seconds, and the period from the beginning of the WC of one leg to the beginning of WC of the other leg (SDS).

Impact loads were assessed at the beginning of support phase, and the parameter "Load" was measured in gs (accelerations from gravity).

Movements in the joints were analyzed as follows (Figure 2): for hip flexion-extension we recorded maximum flexion amplitudes at the beginning of SP (A1, degrees) and the phase of the amplitude (X1, $\%$ of $\mathrm{WC})$ and maximum extension amplitude (A2, degrees) and its phase (X2, \% of WC).
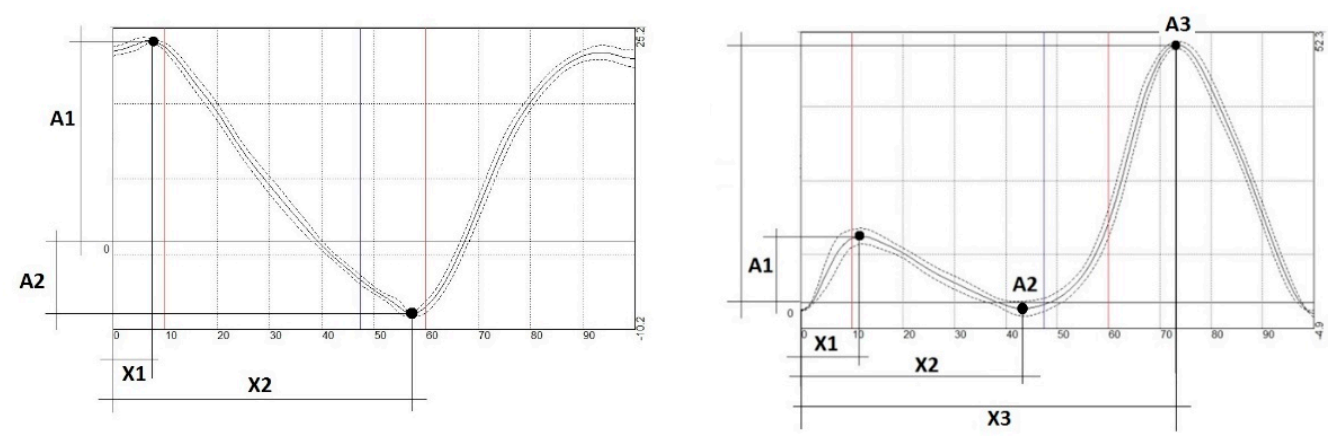

Figure 2. Measured amplitudes (A) and phases (X) in the hip (left graph) and knee (right graph) goniograms.

For knee flexion-extension, we recorded the amplitude of first flexion (A1) and its phase (X1), the amplitude and the phase of extension (A2 and X2, respectively), and the amplitude and phase of second flexion with their maxima in the swing period ( $\mathrm{A} 3$ and $\mathrm{X} 3$ ).

For abduction-adduction and rotation movements of both joints, we recorded the total, maximum amplitude over the WC.

The obtained data were processed using standard methods of ANOVA in Statistica 12 (StatSoft Inc., Tulsa, OK, USA). The means and standard deviations (SDs) were calculated. The significance of differences was assessed using the Wilcoxon-Mann-Whitney test with $p<0.05$. Comparative assessments of the analyzed parameters were done for affected side versus healthy side and versus healthy control.

\section{Results}

As shown by the analysis, the WC and SDS values were similar to those in the control group, being 1.1-1.3 s and 50\%, respectively. Impact loads, however, showed significant changes (see Figure 3). 


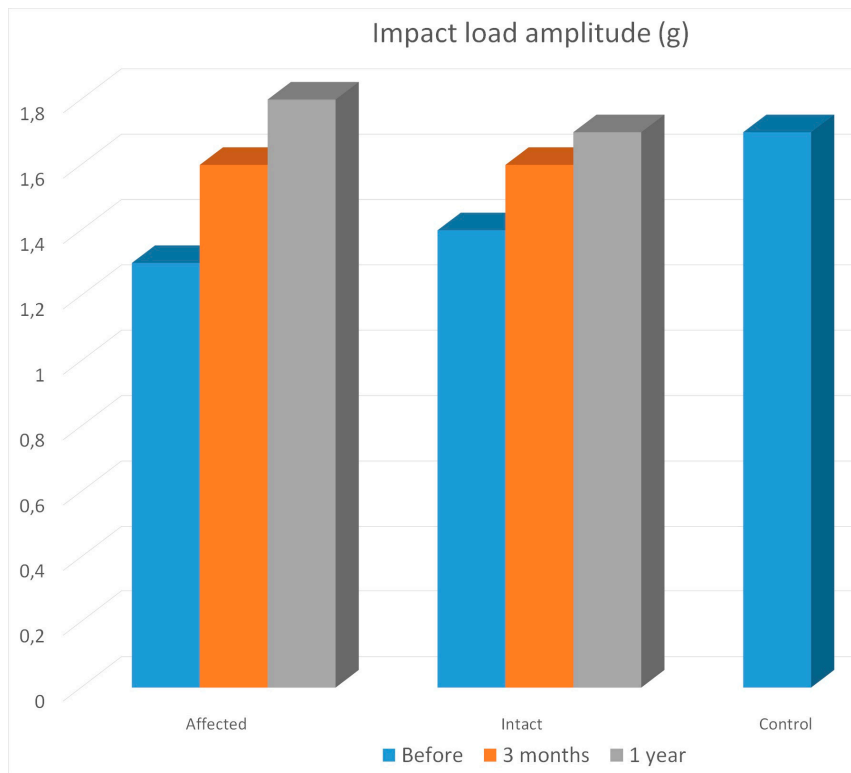

Figure 3. Load amplitudes (g) for the affected and intact legs at different time points (before surgery, three months, and one year or more after ACL reconstruction), compared to the control group.

The magnitude of impact load at the beginning of the stance phase showed the typical dynamics on both sides; it increased with time after surgery. Impact loads were significantly reduced on both sides. Before surgery, impact loads on the intact and affected sides differed significantly from control. At the other time points, impact loads on both sides differed significantly from the respective values before surgery $(\mathrm{p}<0.05)$.

WC duration data are presented in Figure 4.

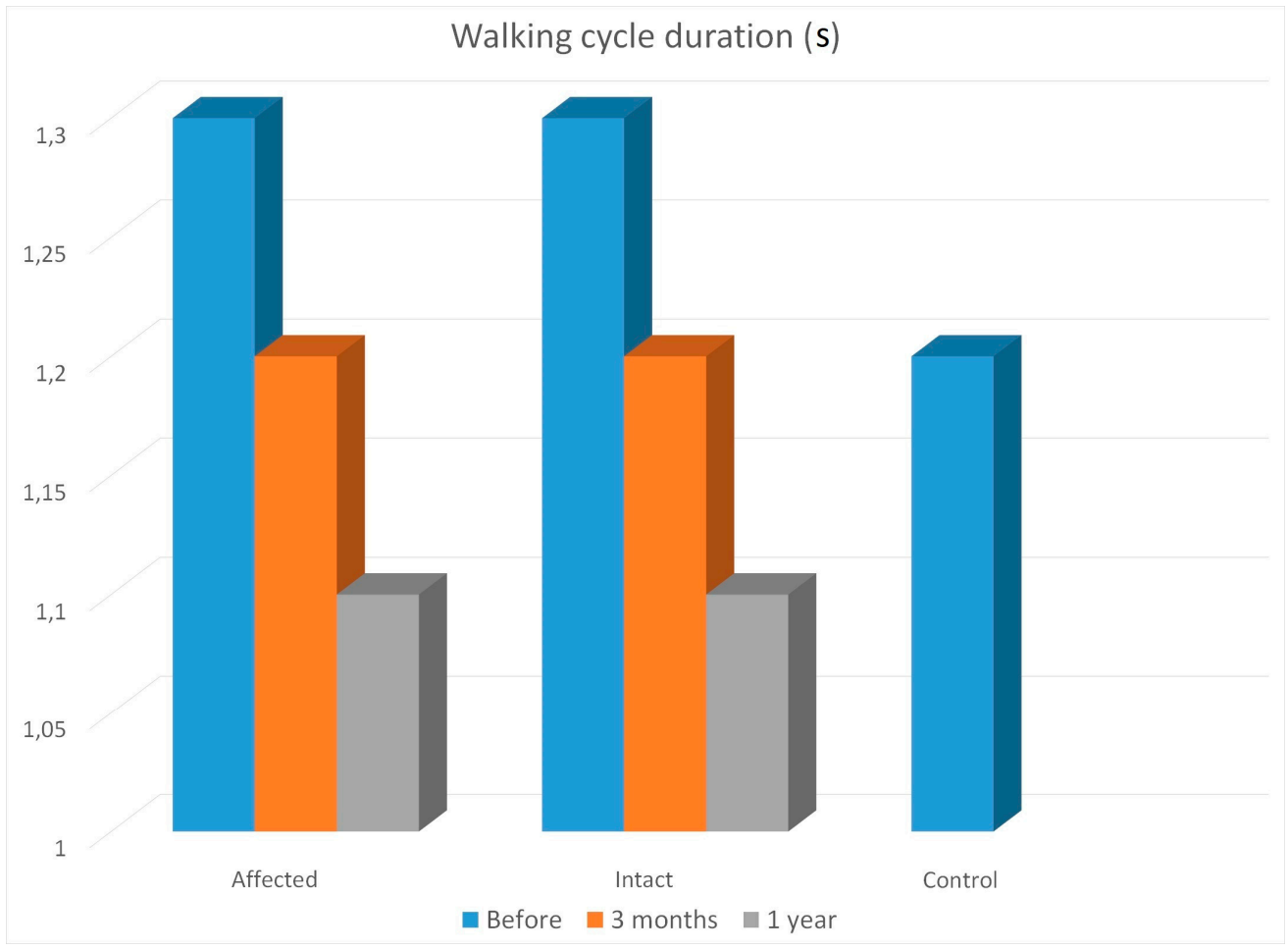

Figure 4. Walking cycle (WC) duration(s) for both sides at different time points, compared to the control group. 
One can see that the WC duration decreased with time after surgery. The changes were not statistically significant, but the trend was obvious. All the values were within normal ranges; however, as the WC duration decreased the walking speed increased, all other things being equal.

The beginning of second double support phase (the parameter SDS) was also within the control range and showed no tendency to change.

The observed changes in hip joint kinematics are presented in Table 2.

Table 2. Hip movement amplitudes.

\begin{tabular}{cccccccc}
\hline \multirow{2}{*}{ Parameter } & \multicolumn{5}{c}{ Affected } & \multicolumn{3}{c}{ Intact } & \multirow{2}{*}{ Control } \\
\cline { 2 - 7 } & Before & 3 months & 1 year & Before & 3 months & 1 year & \\
\hline X1 & $2.9 \pm 1.8$ & $4.0 \pm 2.5^{*}$ & $4.5 \pm 2.2$ & $4.2 \pm 2.0$ & $4.8 \pm 2.9$ & $5.9 \pm 3.0^{\#}$ & $3.8 \pm 2.1$ \\
\hline A1 & $21.1 \pm 7.3^{\#}$ & $19.4 \pm 4.4^{* \#}$ & $20.7 \pm 5.8^{\#}$ & $22.4 \pm 5.6^{\#}$ & $20.7 \pm 5.1^{* \#}$ & $21.1 \pm 5.1^{\#}$ & $26.3 \pm 4.1$ \\
\hline $\mathrm{X} 2$ & $58.2 \pm 3.1^{\#}$ & $58.1 \pm 2.7^{\#}$ & $58.0 \pm 2.2^{\#}$ & $58.6 \pm 4.6^{\#}$ & $57.4 \pm 3.1$ & $57.9 \pm 1.3^{\#}$ & $56.0 \pm 2.1$ \\
\hline A2 & $-7.6 \pm 8.6$ & $-1.3 \pm 5.3^{*}$ & $-13.3 \pm 5.2$ & $-7.9 \pm 7.2$ & $-10.3 \pm 5.0^{*}$ & $-10.8 \pm 6.2$ & $-10.6 \pm 4.2$ \\
\hline Adduction & $11.4 \pm 4.6$ & $12.6 \pm 5.6$ & $15.0 \pm 4.2$ & $13.0 \pm 4.0$ & $13.1 \pm 4.3$ & $18.7 \pm 5.9$ & $13.5 \pm 3.8$ \\
\hline Rotation & $10.7 \pm 3.6$ & $10.9 \pm 3.2$ & $10.9 \pm 1.3$ & $12.0 \pm 4.7$ & $11.4 \pm 3.6$ & $11.0 \pm 2.5$ & $13.1 \pm 5.6$ \\
\hline \multicolumn{7}{c}{ * significant difference from before-surgery value. \# significant difference from the control group. }
\end{tabular}

Before ACL reconstruction, hip movement amplitudes on both sides (affected and unaffected) were significantly smaller than in control $(\mathrm{p}<0.05)$. They remained so in the follow-up periods after the reconstruction. Meanwhile, extension amplitude (A2) increased on the affected side, and abduction-adduction amplitude increased, though insignificantly, on both sides. The moments of maximum flexion and extension ( $\mathrm{X} 1$ and $\mathrm{X} 2$ ) significantly increased (delayed) at different time points of the study. For $\mathrm{X} 2$, the changes were significant compared to control in all study periods ( $\mathrm{p}<0.05$ ).

There were no significant changes in abduction-adduction and rotation movements.

Knee joints showed characteristic changes associated with ACL injury and the subsequent functional restoration (Table 3). The amplitude of knee extension in single support phase was slightly increased and remained so at all times of the study, both on the affected and unaffected sides. The greatest changes were observed in the main amplitude of knee flexion in swing phase (A3). Before ACL reconstruction, the amplitude was significantly reduced on the affected side, by over 10 degrees versus control, and much less so on the intact side. Later on, the amplitude increased gradually, and after a year, it reached, on the operated side, the same values as in the control group. Similar, though less intensive, changes were seen on the intact side.

Table 3. Knee joint movements.

\begin{tabular}{|c|c|c|c|c|c|c|c|}
\hline \multirow{2}{*}{ Parameter } & \multicolumn{3}{|c|}{ Affected } & \multicolumn{3}{|c|}{ Intact } & \multirow{2}{*}{ Control } \\
\hline & Before & 3 months & 1 year & Before & 3 months & 1 year & \\
\hline $\mathrm{X} 1$ & $17.9 \pm 3.4^{\#}$ & $17.8 \pm 2.9$ & $17.3 \pm 3.2$ & $15.4 \pm 2.7^{\#}$ & $15.9 \pm 3.0$ & $16.8 \pm 2.0$ & $16.7 \pm 2.3$ \\
\hline A1 & $13.8 \pm 5.6^{\#}$ & $14.4 \pm 4.7^{\#}$ & $18.7 \pm 4.1$ & $14.5 \pm 4.4^{\#}$ & $16.8 \pm 4.5^{\#}$ & $18.6 \pm 4.9$ & $19.9 \pm 3.8$ \\
\hline $\mathrm{X} 2$ & $37.8 \pm 6.1^{\#}$ & $38.8 \pm 5.7^{\#}$ & $40.9 \pm 5.5$ & $38.7 \pm 6.5^{\#}$ & $39.8 \pm 5.9^{\#}$ & $41.3 \pm 4.1$ & $43.5 \pm 2.2$ \\
\hline $\mathrm{A} 2$ & $8.9 \pm 4.9^{\#}$ & $10.5 \pm 3.9^{\#}$ & $11.2 \pm 3.7^{\#}$ & $7.6 \pm 3.6$ & $10.5 \pm 5.0^{\text {*\# }}$ & $10.1 \pm 5.6^{\#}$ & $6.7 \pm 3.6$ \\
\hline $\mathrm{X} 3$ & $73.8 \pm 4.4$ & $74.2 \pm 2.3$ & $75.4 \pm 1.8^{\#}$ & $76.8 \pm 4.0^{\#}$ & $75.4 \pm 2.5^{\#}$ & $74.2 \pm 1.7$ & $73.9 \pm 1.3$ \\
\hline A3 & $54.2 \pm 17.9^{\#}$ & $59.6 \pm 13.0^{\#}$ & $67.1 \pm 4.7$ & $61.3 \pm 12.6^{\#}$ & $64.0 \pm 8.2^{* \#}$ & $67.8 \pm 6.1$ & $68.7 \pm 5.4$ \\
\hline Adduction & $11.5 \pm 6.0$ & $10.6 \pm 4.1$ & $13.2 \pm 4.0$ & $14.1 \pm 7.3$ & $12.5 \pm 5.0$ & $14.0 \pm 4.9$ & $17.8 \pm 8.3$ \\
\hline Rotation & $13.5 \pm 5.0$ & $14.5 \pm 4.6$ & $15.0 \pm 4.0$ & $18.2 \pm 7.0$ & $19.7 \pm 6.4$ & $16.8 \pm 3.4$ & $21.0 \pm 8.5$ \\
\hline
\end{tabular}


In patients before surgery, both on the affected and unaffected sides, the first knee flexion phase (X1) ended slightly, though significantly, earlier than in the control group $(p<0.05)$. Before and three months after surgery, the extension phase (X2) on both sides (affected and unaffected) ended considerably earlier than in the control group. In the post-operative period, a significant delay of maximum knee flexion in the swing phase (X3) persisted for a year or more on the affected side and up to three months on the intact side.

Abduction-adduction and rotation movements were slightly decreased on both sides and showed no distinct trends.

\section{Discussion}

The results of this study are consistent with earlier findings on the subject $[4,5]$. The age of the patient had no effect on surgery pre- and post-operative management. All the patients adhered to their prescribed treatment. We learnt more about the course of walking function recovery. Some of the studied parameters were shown to be interrelated. For example, the decreased hip flexion-extension amplitude before the reconstruction led to a change in the stride length and, indirectly, to a shorter WC duration. After the ACL surgery the extension improved, albeit insignificantly, and the amplitude increased to reach the control group values 2-3 months after reconstruction, followed by a further slight increase, as was observed a year or more after surgery.

Impact loads also increased linearly up to normal values, with amplitude delta (the difference between its previous and subsequent value) being higher on the affected side. This was an expected result, but we had no opportunity to compare it with other studies. However, the obtained results are fully consistent with the "rule of functional copying" (which states that the healthy limb "copies" the function of the affected one to reduce functional asymmetry) formulated in Skvortsov [11]. The increasing dynamic load at the beginning of the stance phase is in itself the evidence that the existing walking limitations resolve after ACL reconstruction.

ACL injury caused a reduction of hip motion amplitude, which to some extent persisted throughout the study. In addition to the amplitude reduction and its partial recovery due to increased extension, there was a persistent delay of the main extrema of amplitudes. We failed to find any mentioning of this phenomenon in the available literature and we think that further research is needed.

The knee joints showed the largest functional changes. These included reduced amplitudes of first and second flexions as well as reduced extension amplitude. First, flexion was slightly delayed, extension ended earlier, and the main swing flexion (A2) was also slightly delayed compared with the control group. These changes were bilateral, which is again in accordance with the rule of functional copying.

The observed functional changes, as a whole, are specific for the pathology under study and for the subsequent partial recovery in the post-operative period.

The study by Davis et al. [2] also noted that the healthy side developed functional changes similar to those observed on the operated side in the long-term period after ACL reconstruction. At the same time, another study [6] showed functional asymmetries in knee joint kinematics during walking in the long-term (two or more years) after ACL reconstruction. The differences, however, relate to parameters (anteroposterior displacement of the lower leg relative to the thigh, adduction in the knee joint, and torque increase) not assessed in the current study due to its different design and research equipment. Erhart-Hledik et al. [12] rightly observe that functional assessments of walking and of the knee joint should be done as early as possible after ACL reconstruction to detect potential complications such as osteoatritis. The latter can develop in the long term after ACL reconstruction, which is indirectly confirmed by another study [4] that showed decreased movement amplitudes in the operated knee joints eight years or more after surgery. 


\section{Conclusions}

In conclusion, complete functional recovery of the knee joint was not achieved within a year after ACL surgical reconstruction. The remaining changes, however, were not clinically pronounced and could only be detected by instrumental gait analysis. The compensatory processes developed bilaterally, both in the hip and knee joints.

Based on the gradual increase of movement amplitudes from pre-surgery values to those observed three months and a year or more post-surgery, one can extrapolate the process beyond the period of this study and expect a better functional recovery of the operated knee under otherwise equal conditions.

We suppose that objective gait analysis, and especially knee and hip kinematics, should be included in the clinical protocol for ACL rupture patients in the rehabilitation period (after the surgical reconstruction). This is the only study that can evaluate the function of the operated joint and that of the lower limbs as a whole. The results of such function studies are more informative and cannot be obtained by routine clinical assessments.

Author Contributions: D.S. - conceptualization, methodology, formal analysis, writing-original draft preparation, writing-review and editing; S.K. - patient investigation, formal analysis, writing - original draft preparation, writing-review and editing; A.A. (Alexander Akhpashev)- methodology, formal analysis, writing-original draft preparation; A.A. (Aljona Altukhova )- patient investigation, first time analysis of data and data preparation; A.T. data curation, project administration, funding acquisition, supervision; N.Z. - data curation, project administration. All authors have read and agreed to the published version of the manuscript.

Funding: The study was funded as part of the government assignment to FNKC FMBA, state registration No. 20.001.16.800.

Conflicts of Interest: The authors declare no conflict of interest.

\section{References}

1. De Oliveira, E.A.; Andrade, A.O.; Vieira, M.F. Linear and nonlinear measures of gait variability after anterior cruciate ligament reconstruction. J. Electromyogr. Kinesiol. 2019, 46, 21-27. [CrossRef] [PubMed]

2. Davis, K.; Williams, J.L.; Sanford, B.A.; Zucker-Levin, A. Assessing lower extremity coordination and coordination variability in individuals with anterior cruciate ligament reconstruction during walking. Gait Posture 2019, 67, 154-159. [CrossRef] [PubMed]

3. Capin, J.J.; Zarzycki, R.; Arundale, A.; Cummer, K.; Snyder-Mackler, L. Report of the Primary Outcomes for Gait Mechanics in Men of the ACL-SPORTS Trial: Secondary Prevention With and Without Perturbation Training Does Not Restore Gait Symmetry in Men 1 or 2 Years After ACL Reconstruction. Clin. Orthop. Relat. Res. 2017, 475, 2513-2522. [CrossRef] [PubMed]

4. Goetschius, J.; Hertel, J.; Saliba, S.A.; Brockmeier, S.F.; Hart, J.M. Gait Biomechanics in Anterior Cruciate Ligament-reconstructed Knees at Different Time Frames Postsurgery. Med. Sci. Sports Exerc. 2018, 50, 2209-2216. [CrossRef] [PubMed]

5. Majewska, J.; Szczepanik, M.; Szymczyk, D.; Bazarnik-Mucha, K.; Drużbicki, M.; Snela, S.; Jarmuziewicz, A.; Pyczuła, R. Evaluation of Selected Gait Parameters in Patients Prior to and at 6 Months Following Early Anterior Cruciate Ligament Reconstruction. Ortop. Traumatol. Rehabil. 2017, 19, 273-283. [CrossRef] [PubMed]

6. Erhart-Hledik, J.C.; Chu, C.R.; Asay, J.L.; Andriacchi, T.P. Longitudinal changes in knee gait mechanics between 2 and 8 years after anterior cruciate ligament reconstruction. J. Orthop. Res. 2018, 36, 1478-1486. [CrossRef] [PubMed]

7. Slater, L.V.; Hart, J.M.; Kelly, A.R.; Kuenze, C.M. Progressive Changes in Walking Kinematics and Kinetics After Anterior Cruciate Ligament Injury and Reconstruction: A Review and Meta-Analysis. J. Athl. Train. 2017, 52, 847-860. [CrossRef] [PubMed]

8. Milandri, G.; Posthumus, M.; Small, T.J.; Bothma, A.; van der Merwe, W.; Kassanjee, R.; Sivarasu, S. Kinematic and kinetic gait deviations in males long after anterior cruciate ligament reconstruction. Clin Biomech. 2017, 49, 78-84. [CrossRef] [PubMed] 
9. Shi, H.; Huang, H.; Ren, S.; Yu, Y.; Liang, Z.; Wang, Q.; Hu, X.; Ao, Y. The relationship between quadriceps strength asymmetry and knee biomechanics asymmetry during walking in individuals with anterior cruciate ligament reconstruction. Gait Posture 2019, 73, 74-79. [CrossRef] [PubMed]

10. Skvortsov, D.; Kaurkin, S.; Goncharov, E.; Akhpashev, A. Knee joint function and walking biomechanics in patients in acute phase anterior cruciate ligament tear. Int. Orthop. 2020. [CrossRef] [PubMed]

11. Skvortsov, D.V. Diagnosis of motor pathology with instrumental methods: Gait analysis, stabilometry. Nauch.-med. firma MBN. 2007, p. 640. Available online: https://rehabrus.ru/Docs/Diagn_dvig_patalogii_ 2007.pdf (accessed on 20 April 2018). (in Russian).

12. Erhart-Hledik, J.C.; Chu, C.R.; Asay, J.L.; Andriacchi, T.P. Gait mechanics 2 years after anterior cruciate ligament reconstruction are associated with longer-term changes in patient-reported outcomes. J. Orthop. Res. 2017, 35, 634-640. [CrossRef] [PubMed]

(C) 2020 by the authors. Licensee MDPI, Basel, Switzerland. This article is an open access article distributed under the terms and conditions of the Creative Commons Attribution (CC BY) license (http://creativecommons.org/licenses/by/4.0/). 